\title{
AN EXPERIMENTAL INVESTIGATION INTO THE SCALE-UP OF OSCILLATORY FLOW MIXING IN BAFFLED TUBES
}

\author{
K. B. SMITH and M. R. MACKLEY* \\ Department of Chemical Engineering, University of Cambridge, UK
}

\begin{abstract}
$\mathrm{T}$
his paper describes results from an experimental investigation into the scale-up of oscillatory flow mixing (OFM) in geometrically scaled baffled tubes ranging from $24 \mathrm{~mm}$ to $150 \mathrm{~mm}$ diameter and from $1 \mathrm{~m}$ to $4.5 \mathrm{~m}$ in length. Measured axial dispersion coefficients are reported together with case studies and an empirical correlation. An alternative multi-orifice reactor geometry with the potential for use in large-scale plant was also constructed and tested from which it was concluded that OFM is a technology which lends itself readily to scaling-up from laboratory to pilot plant scale, and to industrial scale. Experiments performed on small laboratory OFM apparatus containing less than one litre of fluid can with confidence be used to predict mixing behaviour in much larger plant containing hundreds of litres of fluid.
\end{abstract}

Keywords: oscillatory flow; mixing; axial dispersion; flow visualization; scale-up.

\section{INTRODUCTION}

It has often been the goal of chemical engineers to construct a reactor design that would give perfect plug flow, even under variable throughput conditions. Tubular reactors are a close approximation, yet in general (a) are reliant on turbulent net flow, (b) are susceptible to variations in throughput and (c) can for long residence times require very long tubes with resulting high pressure differences along the length of the reactor. Oscillatory Flow Mixing (OFM) in baffled tubes has previously been shown to have a number of advantageous properties at laboratory scale (Mackley, 1987, 1991). Most notably, control of the oscillatory conditions when operating as a continuous process allows axial dispersion to be minimised (Dickens et al., 1989), permitting the control of residence time distributions independently of the throughput rate as shown by (Mackley and Ni, 1991) so that the technology can be operated as a near-perfect plug flow device even with variable throughput.

OFM typically consists of periodically spaced annular baffles inside a long tube in which either a liquid or a multiphase mixture is oscillated axially (Brunold et al., 1989). This induces vortices on both sides of the baffle which provide both axial and radial mixing in the tube and imparts a unique form of control of mixing and transfer processes in the tube. The intensity of mixing can be varied by tuning the oscillatory conditions (amplitude and frequency of

* Correspondence to: Professor M. R. Mackley, Department of Chemical Engineering, University of Cambridge, Pembroke Street, Cambridge, CB2 3RA, UK.

E-mail: mrm5@cam.ac.uk oscillation) and Howes (1988) identified several different mixing regimes ranging from creeping laminar to fully turbulent flow. Previous OFM studies such as control of residence time distribution, improved heat transfer (Mackley and Stonestreet, 1995) and particle suspension (Mackley et al., 1993) have in general been limited to laboratory scale with only a few litres of liquid in the reactor volume. This paper therefore seeks to understand more fully the effect of scale-up on the performance of OFM. The paper reports a programme of experiments on geometrically scaled apparatus with the measurement of residence time distributions as the principal method of investigating the wide range of flow conditions that can be achieved by control of net flow and of oscillatory conditions. Results from these investigations are interpreted as axial dispersion coefficients, knowledge of which enables the prediction of bulk mixing times (batch systems) or of residence time distributions (continuous systems).

\section{DIMENSIONLESS GROUPS}

Previous studies of OFM have adopted the following dimensionless groups to describe flow conditions in the baffled tube, assuming an approximately sinusoidal driving oscillation (Ni and Gough, 1997):

Net flow Reynolds number

$$
R e_{\mathrm{n}}=\frac{\rho U d}{\mu}
$$


Oscillatory Reynolds number

$$
R e_{\mathrm{o}}=\frac{\rho \omega x_{\mathrm{o}} d}{\mu}
$$

Strouhal number

$$
S t r=\frac{d}{4 \pi x_{\mathrm{o}}}
$$

In additional, the following dimensionless groups have been adopted to quantify axial dispersion:

Peclet number

$$
P e=\frac{U L}{E}
$$

Schmidt number

$$
S c=\frac{\mu}{\rho E}
$$

where $d$ is the tube internal diameter, $E$ is axial dispersion coefficient, $L$ is length of tube, $U$ is mean net flow velocity through tube, $x_{\mathrm{o}}$ is oscillatory amplitude (centre to peak), $\mu$ is viscosity of fluid, $\rho$ is density of fluid, $\omega_{\mathrm{o}}$ is angular velocity of oscillation.

The Strouhal number Str is therefore inversely proportional to the amplitude of oscillation, and together with $R e_{\mathrm{o}}$ and $R e_{\mathrm{n}}$ are assumed in the literature to define fully the fluid dynamic conditions for a particular geometry. The Peclet $P e$ and Schmidt $S c$ numbers are both inversely proportional to the axial dispersion coefficient; the Peclet number is also related to the physical geometry and flow conditions of the experiment and is therefore of use when gauging the likely magnitude of experimental errors or the effectiveness of a particular reactor, whereas the Schmidt number is related to the fundamental properties of the fluid and therefore is relevant for the consideration of scale-up.

\section{APPARATUS}

Three sets of geometrically similar apparatus were designed and constructed (Smith, 1999). The smallest apparatus was a $1 \mathrm{~m}$ tall tube of $24 \mathrm{~mm}$ internal diameter, the intermediate was a $2 \mathrm{~m}$ tall tube of $54 \mathrm{~mm}$ internal diameter (Figure 1) and the largest was a $4.5 \mathrm{~m}$ tall tube of $150 \mathrm{~mm}$ internal diameter comparable in size to industrial pilot-scale plant. Each apparatus contained single orifice (or annular) baffles, spaced at intervals of 1.5 tube diameters apart along the tube. Sinusoidal oscillation of the fluid was by a servo-hydraulic cylinder and piston arrangement at the base of the tube and the fluid level inside the tube was controlled by an outlet overflow port. Each apparatus can be operated either in batch mode (simply a mixing device) or in continuous mode with a net flow of fluid along the tube. Oscillations of $1 \mathrm{~Hz}$ and $1 \mathrm{~mm}$ centre-to-peak (c.t.p.) amplitude and a net flow rate of $120 \mathrm{ml} \mathrm{min}{ }^{-1}$ were taken as a reference point for a typical experiment in the $24 \mathrm{~mm}$ diameter apparatus. When considering an approximate six-fold increase of tube diameter up to $150 \mathrm{~mm}$, in order to keep the Strouhal number constant requires a six-fold increase in c.t.p. amplitude i.e., from $1 \mathrm{~mm}$ to $6 \mathrm{~mm}$; in order to keep the Oscillatory Reynolds number constant requires a thirtysix-fold (i.e., six-squared) decrease in oscillatory frequency i.e., from $1 \mathrm{~Hz}$ to $0.0278 \mathrm{~Hz}$; in order to keep the Net Flow Reynolds number constant the net flow rate through the tube would increase by a factor of six i.e., from $120 \mathrm{ml} \mathrm{min}^{-1}$ to $720 \mathrm{ml} \mathrm{min}{ }^{-1}$.

The inverse-square relationship between tube diameter and oscillation frequency for comparable experiments was considered to be a limiting factor governing the maximum tube diameter $(150 \mathrm{~mm})$ which could sensibly be investigated in the laboratory. Table 1 shows the dimensions and operating ranges of the apparatus.

The Strouhal number Str could be varied for each apparatus from 0.25 (limited by stroke of oscillation) to 8 (above which the oscillation was too small to have a measurable effect on axial dispersion). The oscillatory Reynolds number $R e_{\mathrm{o}}$ could be varied from approximately 0 (no oscillation) to 1200 (mechanical limit of $24 \mathrm{~mm}$ apparatus) or 8500 (limit of $54 \mathrm{~mm}$ apparatus) or 56500 (limit of $150 \mathrm{~mm}$ apparatus). The net flow Reynolds number $R e_{\mathrm{n}}$ could be varied for each apparatus from 0 (no flow) to 3000 for the $24 \mathrm{~mm}$ and $150 \mathrm{~mm}$ apparatus and from 0 to 2000 for the $54 \mathrm{~mm}$ apparatus. These ranges are summarized in Table 2 .

Baffles were machined from $1 \mathrm{~mm}, 2 \mathrm{~mm}$ and $6 \mathrm{~mm}$ thick PVC sheeting for the three rigs respectively and coupled using stainless steel studding. By machining many baffles sandwiched together, a very clean orifice edge could be obtained and also the precise outer diameter could be custom-made to push-fit against the particular tube wall. The baffles have a spacing equivalent to three tube radii and a relatively high constriction ratio of $75 \%$ by area. This constriction is probably greater than the optimum but was chosen to match previous studies in residence time distribution and heat transfer (Stonestreet, 1997) as well as to match reciprocating plate studies performed by (Baird and Rama Rao, 1996). A portion of each apparatus was surrounded by a square viewing box that served to reduce the effect of optical refraction. The operating fluid for all experiments was water. Net flow was regulated by a needle valve and monitored by a range of rotameters (selected according the flow-rate). This gave a steady flow, with the water supply being delivered from headertanks maintained at constant pressure.

\section{DETERMINATION OF AXIAL DISPERSION}

The measurement of axial dispersion for a range of process devices has been extensively studied by a number of authors from which a range of protocols have been developed, see for example (Levenspiel and Smith, 1957) and is also discussed in (Handbook of Solvent Extraction, 1983).

In order to measure axial dispersion an established imperfect pulse technique was used: a pulse of neutrallybuoyant tracer is injected into the tube and then the concentration of tracer is measured at two points downstream. The sensor readings are transposed into concentrations and then normalized so that for example $C_{2}{ }^{\prime}$ and $C_{1}{ }^{\prime}$ are 


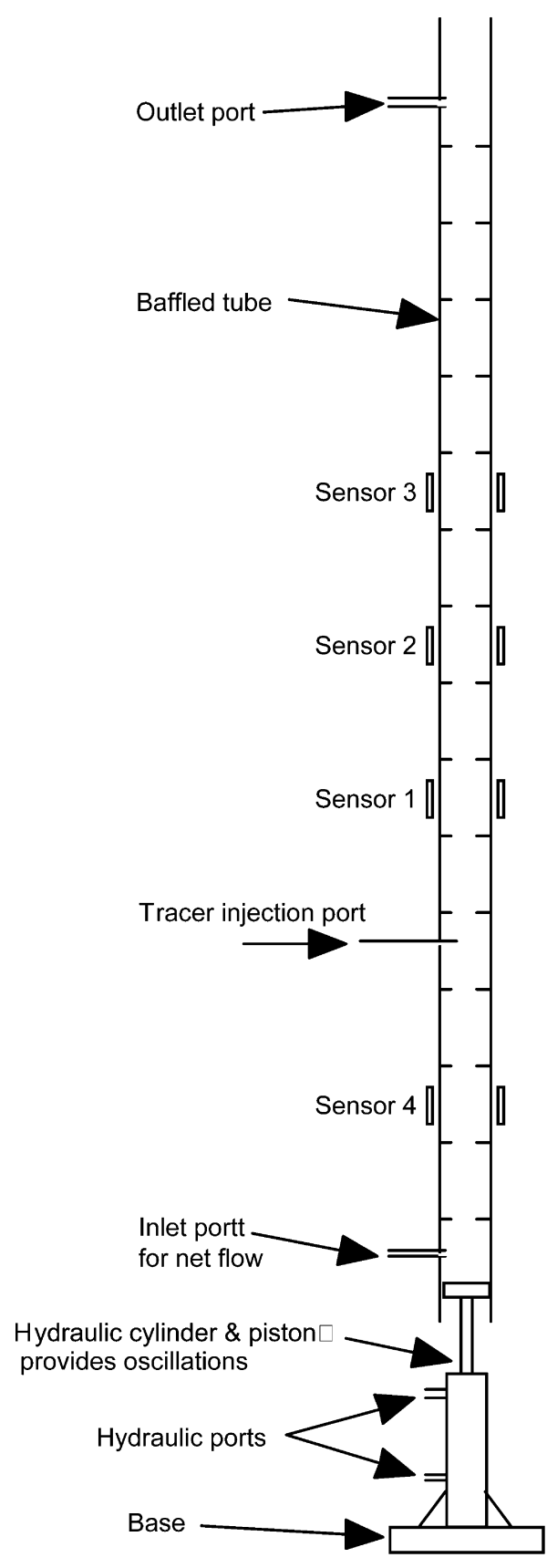

(a)

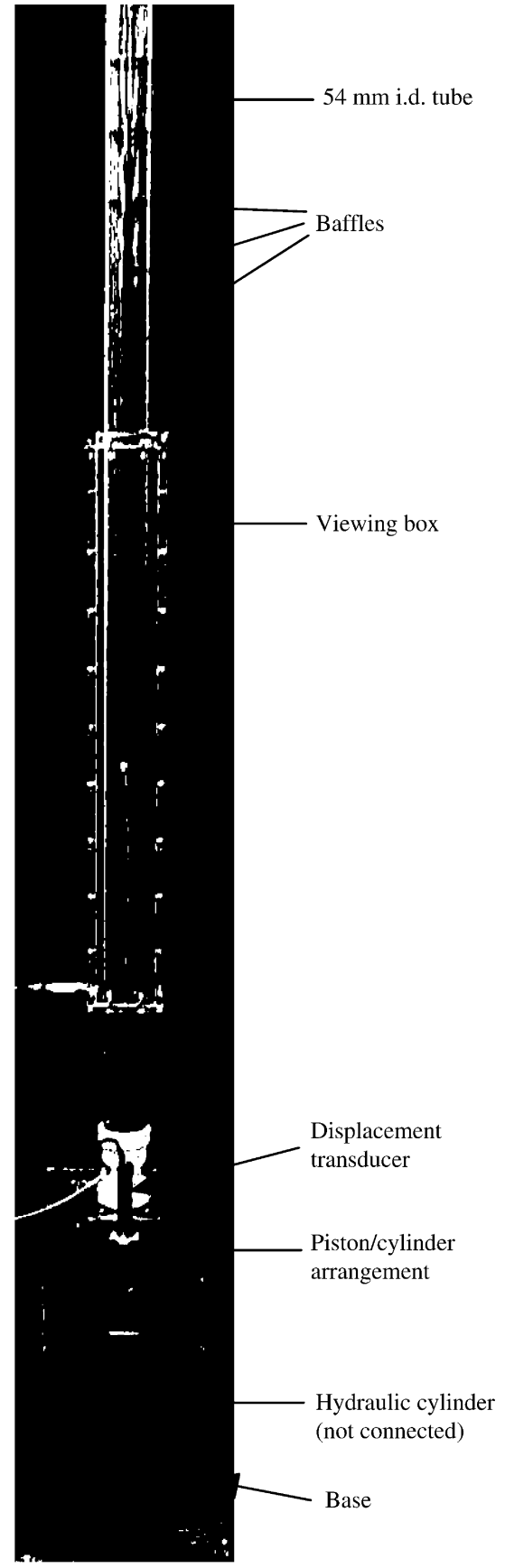

(b)

Figure 1. (a) Schematic diagram of oscillatory flow mixing apparatus. (b) Photograph of the 54 mm apparatus with its viewing box.

the normalized experimental concentrations at optical sensors 2 and 1 respectively:

$$
C_{2}^{\prime}(t)=\frac{C_{2}(t)}{\int_{0}^{\infty} C_{2}(t) d t}
$$

and

$$
C_{1}^{\prime}(t)=\frac{C_{1}(t)}{\int_{0}^{\infty} C_{1}(t) d t}
$$

The normalization is not strictly necessary, but allows the results to be scaled conveniently onto a single axes for comparisons to be made. In the case of experiments without net flow, the normalization procedure cannot be applied and therefore the data is retained as a concentration in $\mathrm{mg}^{-1}$. Concentration values were averaged over one oscillatory cycle.

The value of the mean net flow velocity $U$ was calculated directly from the net volumetric flow rate $Q$, the distance between the probes $L$ and the volume of fluid between probes $V$ :

$$
U=\frac{Q L}{V}
$$


Table 1. Apparatus dimensions and operating ranges.

Tube diameter, $\mathrm{mm} \quad$ Tube height, $\mathrm{m} \quad$ Tube volume, $\mathrm{m}^{3} \quad$ Range of c.t.p. amplitude, $\mathrm{mm} \quad$ Range of frequency, Hz Range of net flow, ml min ${ }^{-1}$

\begin{tabular}{rlclr}
\hline 24 & 1 & 0.45 & $0-8$ & $0.1-20$ \\
54 & 2 & 4.6 & $0-25$ & $0.05-10$ \\
150 & 4.5 & 79.5 & $0-60$ & $0.01-10$
\end{tabular}

A model (in this work the diffusion equation) is then used to calculate a residence time distribution between the two measuring points based upon their distance apart, the mean net flow velocity of the fluid in the tube and one or more other parameters (Gilibaro, 1978). This model residence time distribution or transfer function is then convoluted with the concentration-time profile measured at the first measuring point in order to predict a concentration-time profile at the second measuring point; the experimental and model concentration-time profiles are then compared.

$$
\begin{aligned}
\text { Transfer function }(t)= & {\left[\frac{1}{\sqrt{4 \pi E \frac{t^{3}}{x^{2}}}}\right] } \\
& \times \exp \left\{-\frac{(x-U t)^{2}}{4 E t}\right\} \\
& \text { Westerterp et al. (1984) }
\end{aligned}
$$

An iterative subroutine is used to determine the value of the axial dispersion coefficient $E$ which caused the model prediction to match most closely the experimental measurements. Although subroutines were developed to optimize $E$ automatically, these were found to be slow as well as insensitive to anomalous data; with a little experience, a process of visual inspection and guessed values of $E$ was found to be the quickest and most informative method of analysing the data to obtain an estimate of $E \pm 10 \%$.

The minimum number of measuring points using the imperfect pulse technique is therefore two, but in practice it is desirable to have at least three measuring points, from which three separate estimates of axial dispersion within the same experiment can be made. This serves as a useful check that conditions are consistent along the tube. Three optical sensors were placed along the tube downstream of the injection point, as well as an additional sensor upstream of the injection point whose purpose was to obtain a measure of the backmixing in the system (i.e., the degree to which the oscillations forced dye tracer upstream against the direction of mean net flow).

Further details of the experimental tracer measurements and data analysis are described in (Smith, 1999); the dye

Table 2. Approximate range of experimental conditions expressed as dimensionless groups.

\begin{tabular}{rccc}
\hline Tube diameter, $\mathrm{mm}$ & $S t r$ & \multicolumn{1}{c}{$R e_{\mathrm{o}}$} & $R e_{\mathrm{n}}$ \\
\hline 24 & $0.25-8$ & $0-1200$ & $0-3000$ \\
54 & $0.25-8$ & $0-8500$ & $0-2000$ \\
150 & $0.25-8$ & $0-56500$ & $0-3000$
\end{tabular}

selected was methylene blue because of its high optical density at low concentrations.

\section{Case Study 1: Axial Dispersion in OFM with Net Flow}

Typical concentration-time profiles for an experiment in the $24 \mathrm{~mm}$ baffled tube are shown in Figure 2. The mean

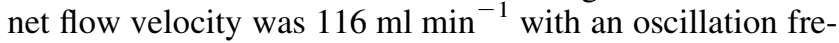
quency of $0.5 \mathrm{~Hz}$ and a centre-to-peak amplitude of $8 \mathrm{~mm}$. Note that the area under the concentration-time profile for the upstream sensor (4) was measured to be about $34 \%$ of that for the other sensors, a measure of the degree of backmixing (axial dispersion of the dye against the direction of net flow). The discrepancy in the total area under the concentration-time profiles for sensors 2 and 3 compared with sensor 1 was $2.1 \%$ and $2.8 \%$ before normalization. This is a relatively small discrepancy within the expected experimental error for the calibrated optical sensors; it supports the observation that effectively all the dye had been successfully flushed through the tube.

Using the transfer function after (Westerterp et al., 1984) gave an excellent agreement between the 'experimental' and predicted 'model' results and an example is shown in Figure 3 for two of the sensors. The model prediction coincides very well with the experimental profile for a consistent value of $E=0.0003 \mathrm{~m}^{2} \mathrm{~s}^{-1}$ for all three pairs of sensors.

\section{Case Study 2: Axial Dispersion in OFM with No Net Flow}

Without net flow (but with oscillations) the dye simply spreads out in near-Gaussian fashion from the injection

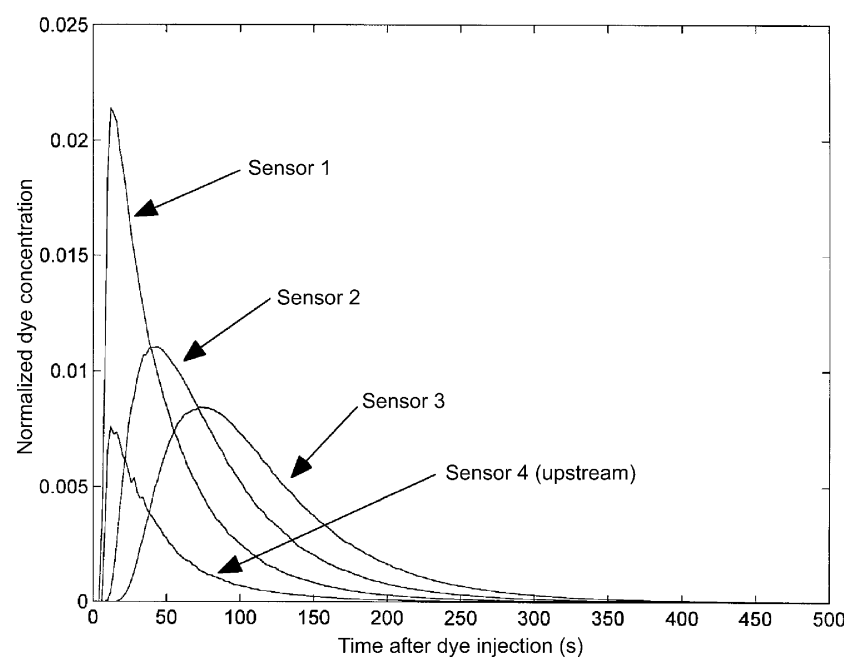

Figure 2. Case study 1: concentration-time profiles for the optical sensors in OFM with net flow. 


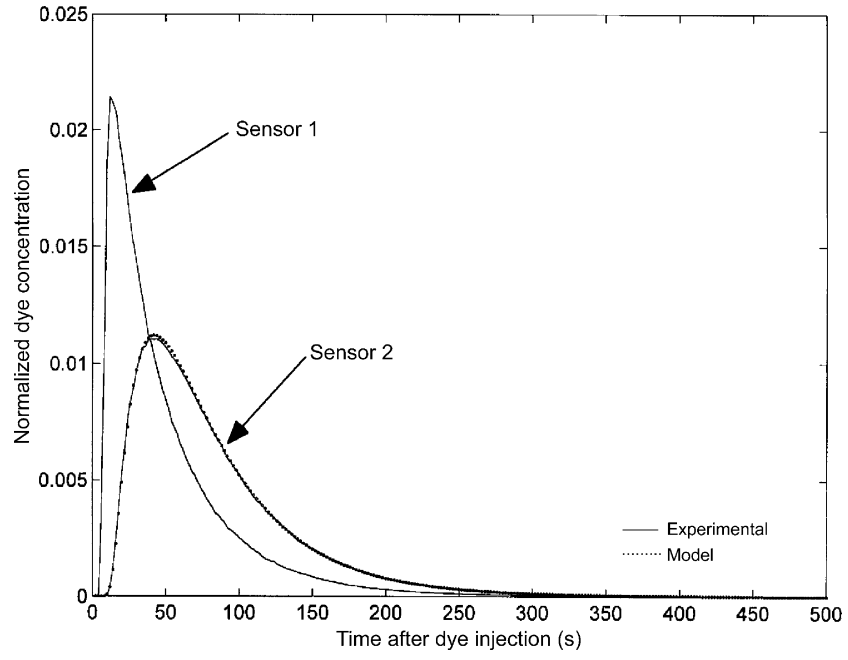

Figure 3. Case study 1: experimental and model results for sensors $1 \rightarrow 2$.

point and remains in the tube. The concentration-time profiles for a typical no net flow experiment in the $150 \mathrm{~mm}$ apparatus with frequency $0.2 \mathrm{~Hz}$ and amplitude $27.8 \mathrm{~mm} \quad\left(R e_{\mathrm{o}}=5300\right.$ and $\left.R e_{\mathrm{n}}=0\right)$ are shown in Figure 4. The analysis used for the imperfect pulse experiments still holds true for the initial changes in concentration following dye injection; only when the dye reached the ends of the baffled tube does the model (which assumed an infinitely long tube) start to differ from the experimental reality. Therefore the initial concentration transients measured immediately after dye injection were of primary interest in order to determine axial dispersion. The analysis routine for iteratively determining $E$ was carried-out in the same manner as for a net-flow experiment, except that the primary concern was to match the initial transient behaviour of the concentration-time profile. Figure 5 shows a good fit between model and experiment for the first $100 \mathrm{~s}$ but thereafter end effects of the tube dominate as expected. A best-fit was obtained for $E=0.0028 \mathrm{~m}^{2} \mathrm{~s}^{-1}$.

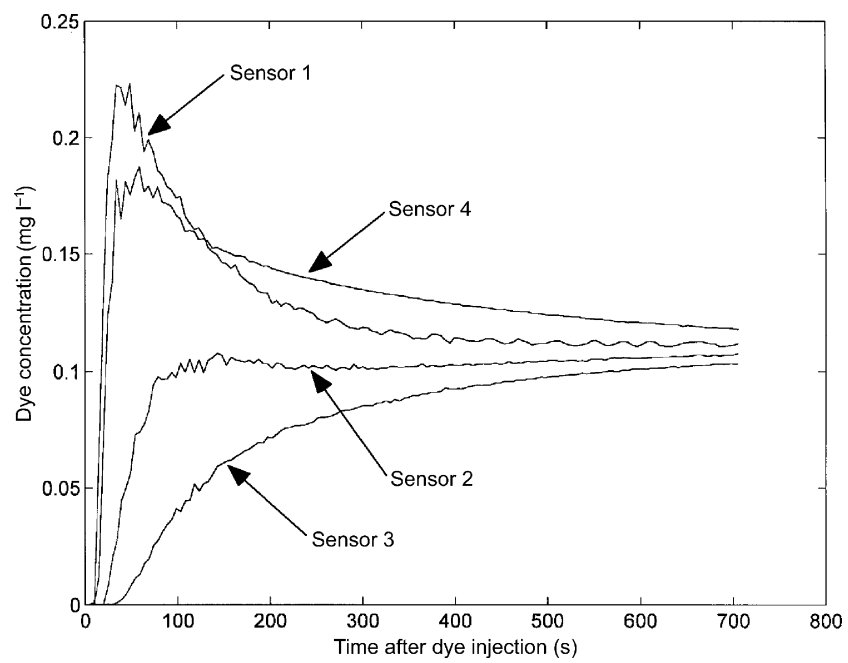

Figure 4. Case study 2: typical concentration-time profiles with no net flow.

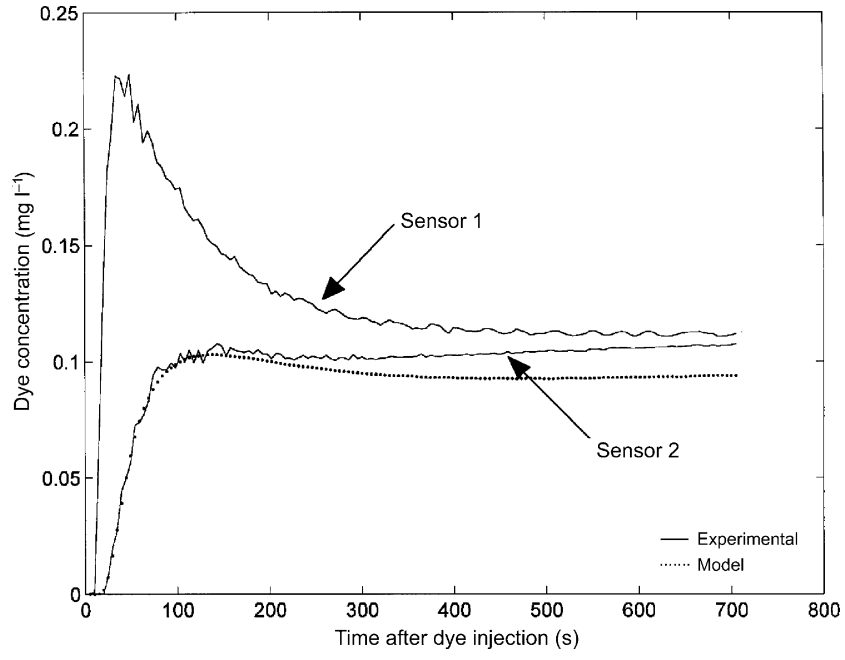

Figure 5. Case study 2: experimental and model results for sensors $1 \rightarrow 2$.

\section{RESULTS}

Experimental axial dispersion measurements were made in each set of apparatus for a large range of flow conditions. The main objective of these experiments was to compare the experimentally measured axial dispersion under dynamically similar flow conditions as a function of tube diameter, a task not previously attempted and of direct importance in terms of scale-up considerations. The overall error for each data point is estimated to be of order $\pm 10 \%$ for axial dispersion and $\pm 5 \%$ for oscillatory Reynolds number; for clarity, error bars are not included on these graphs.

\section{Oscillation with Net Flow}

Figure 6 shows data from all three sets of apparatus with a fixed net flow Reynolds number. Three distinct regimes can be identified: firstly, for $R e_{\mathrm{o}}<80$ the axial dispersion appears to tend to a constant value of approximately $5 \times 10^{-4} \mathrm{~m}^{2} \mathrm{~s}^{-1}$. Secondly, there is a characteristic

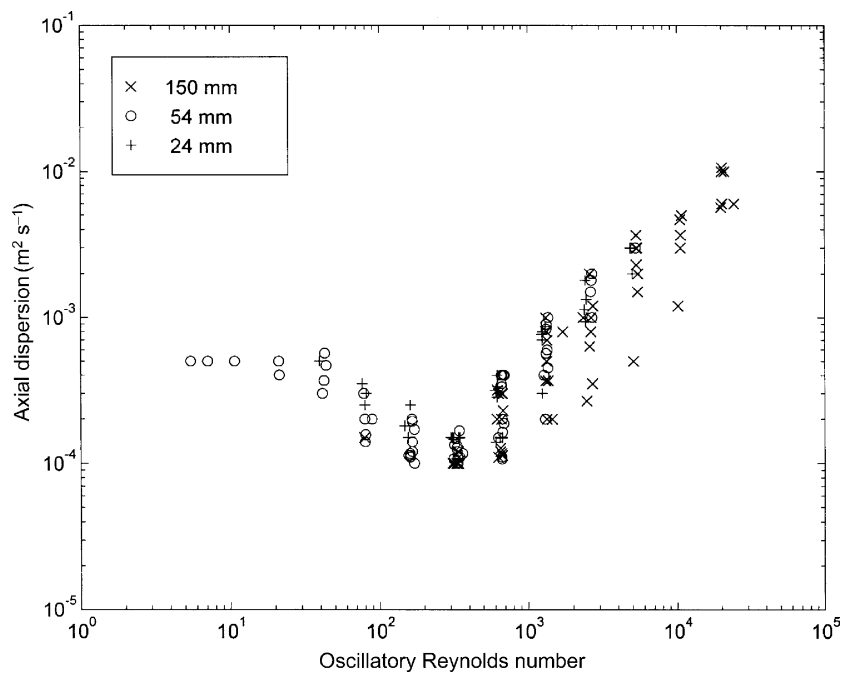

Figure 6. $E$ versus $R e_{\mathrm{o}}$ in the $24 \mathrm{~mm}, 54 \mathrm{~mm}$ and $150 \mathrm{~mm}$ apparatus; $R e_{\mathrm{n}}=107$. 
minimum in axial dispersion for $80<R e_{\mathrm{o}}<800$ similar in form to that observed by Dickens et al. (1989) and with values as low as $10^{-4} \mathrm{~m}^{2} \mathrm{~s}^{-1}$. Thirdly, for $R e_{\mathrm{o}}>800$ the axial dispersion increases approximately linearly with increasing $R e_{\mathrm{o}}$. There is a significant variation in the magnitude of the axial dispersion for a given $R e_{\mathrm{o}}$. Figure 7 plots data taken from the previous figure but shows only those results which correspond to $S t r=0.5$. There is a remarkably good match in experimental axial dispersion values for the three sets of apparatus for a given $R e_{\mathrm{o}}$. Similar data is plotted in Figure 8 for $S t r=2$. Again, there is excellent agreement of axial dispersion values for the three sets of apparatus and which exhibit a characteristic minimum in axial dispersion at approximately $R e_{\mathrm{o}}=300$. The minimum is a result of the interaction between the net flow and oscillatory flow components: at the minimum, the vortices created by the net flow and oscillations are optimized to redistribute the tracer radially across the baffled tube with each oscillatory cycle and hence minimize the axial dispersion. If the oscillations are increased then they serve to increase the axial as well as the radial dispersion and hence the oscillations become the dominant mechanism for axial dispersion. If the oscillations are reduced then the efficacy of radial mixing is reduced and eventually the net flow becomes the dominant mechanism for axial dispersion.

The range of $R e_{\mathrm{o}}$ that could be investigated was restricted for large $R e_{\mathrm{o}}$ by the apparatus itself (limitations of oscillator power meant that the sinusoidal wave form could not be maintained) and for low $R e_{\mathrm{o}}$ by the validity of the model fit obtained using the diffusion model. The range of Strouhal number investigated was limited for very large amplitude oscillations $(S t r<0.25)$ since they exceeded the stroke-length of the apparatus. It was also found that for Str $>8$ in all sets of apparatus the diffusion model was generally inadequate. For OFM. in baffled tubes under dynamically similar flow conditions the absolute value (in $\mathrm{m}^{2} \mathrm{~s}^{-1}$ ) of the experimentally measured axial dispersion appears to be independent of tube diameter.

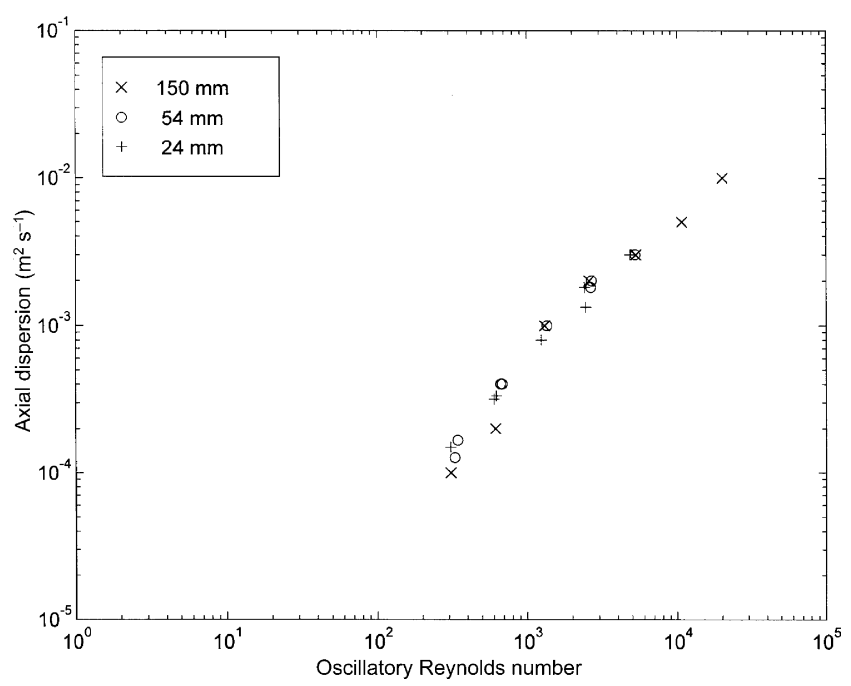

Figure 7. $E$ versus $R e_{\mathrm{o}}$ in the $24 \mathrm{~mm}, 54 \mathrm{~mm}$ and $150 \mathrm{~mm}$ apparatus; $R e_{\mathrm{n}}=107$ and $\operatorname{Str}=0.5$.

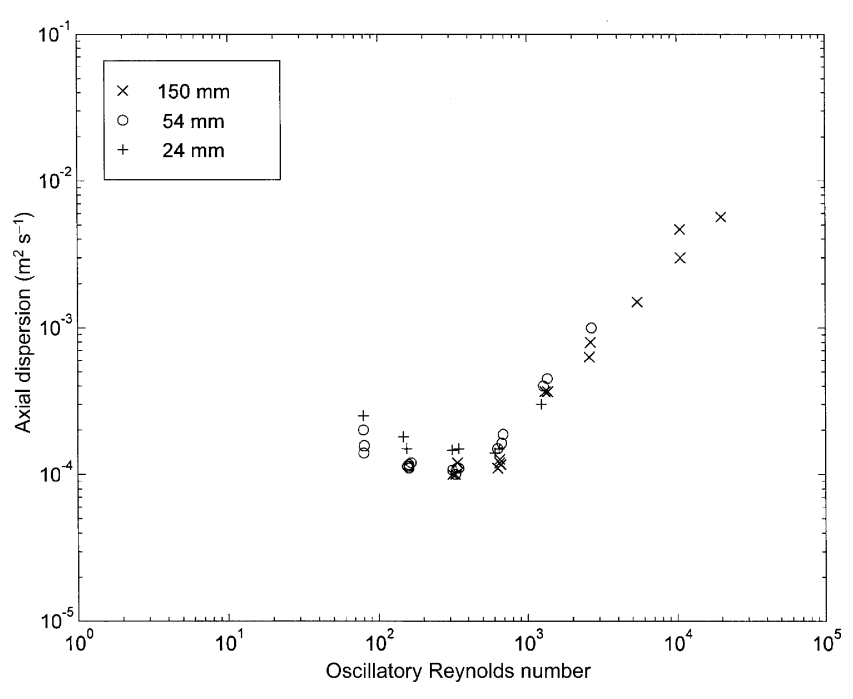

Figure 8. $E$ versus $R e_{\mathrm{o}}$ in the $24 \mathrm{~mm}, 54 \mathrm{~mm}$ and $150 \mathrm{~mm}$ apparatus; $R e_{\mathrm{n}}=107$ and $\operatorname{Str}=2$.

\section{Oscillation with No Net Flow}

Figure 9 shows the measured axial dispersion as a function of $R e_{\mathrm{o}}$. Similar to the previous case, the results show a range of values for a given $R e_{\mathrm{o}}$. Figure 10 shows the same data but only those with a specific Strouhal number $(S t r=1)$. These results again show that the absolute value of experimentally measured axial dispersion in batch OFM is independent of tube diameter. For $S t r \leq 2$ and $R e_{\mathrm{o}}>100$, for a given Str the axial dispersion increases proportionally with oscillation frequency. Decreasing the Strouhal number for a given $R e_{\mathrm{o}}$ increases the magnitude of the axial dispersion. For Str $>2$ there are few results (mostly, the diffusion model gave a very poor fit and therefore the axial dispersion could not be quantified using the imperfect pulse technique). For those results which did fit the diffusion model at high Strouhal numbers, exceptionally low values of axial dispersion

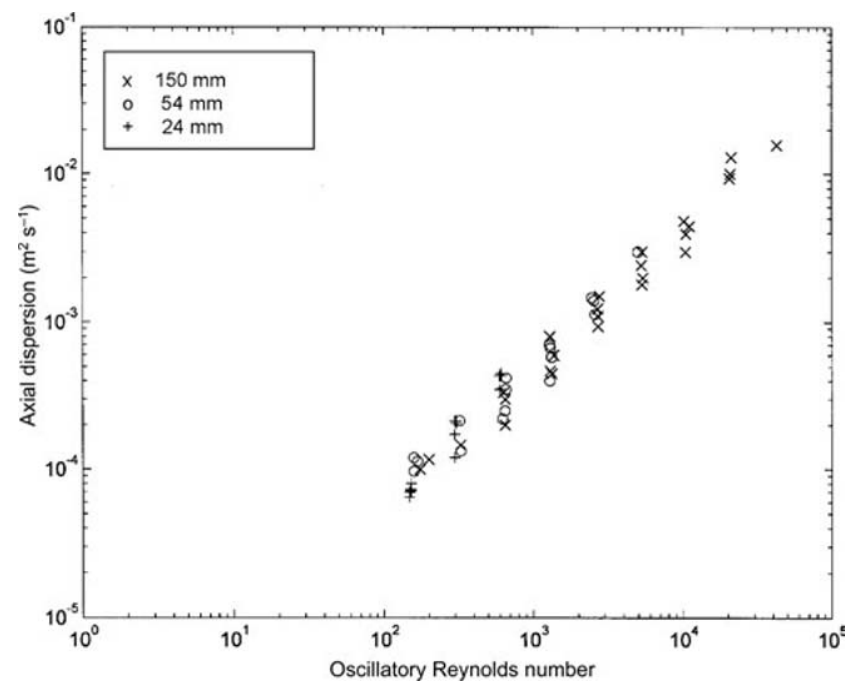

Figure 9. $E$ versus $R e_{\mathrm{o}}$ in the $24 \mathrm{~mm}, 54 \mathrm{~mm}$ and $150 \mathrm{~mm}$ apparatus; oscillation only $R e_{\mathrm{n}}=0$. 


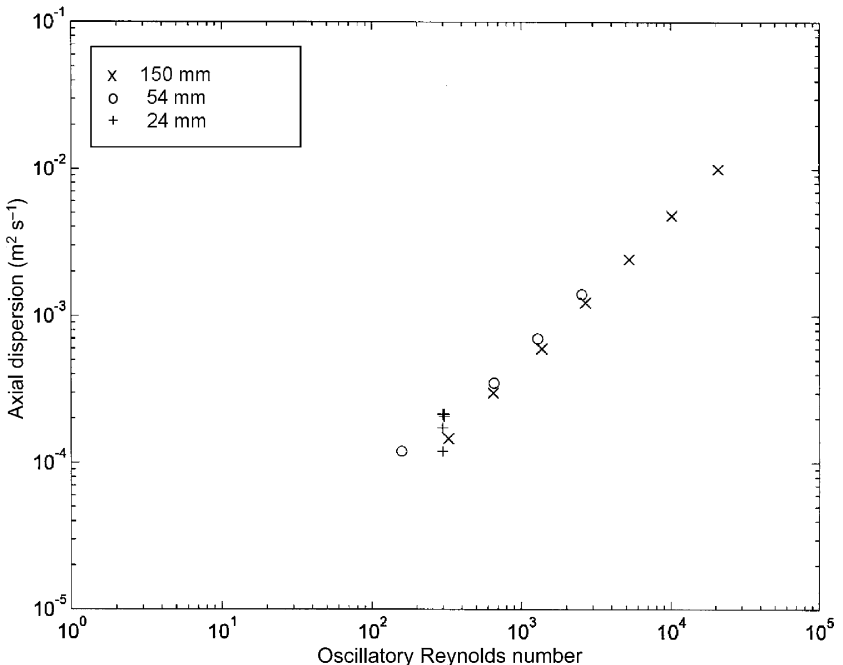

Figure 10. $E$ versus $R e_{\mathrm{o}}$ in the $24 \mathrm{~mm}, 54 \mathrm{~mm}$ and $150 \mathrm{~mm}$ apparatus; $R e_{\mathrm{n}}=0 ; \operatorname{Str}=1$.

were measured: the lowest recorded value was $2.5 \times 10^{-5} \mathrm{~m}^{2} \mathrm{~s}^{-1}$. However, such a result is exceptional and more typically the minimum value of the axial dispersion measured by experiment was of order $10^{-4} \mathrm{~m}^{2} \mathrm{~s}^{-1}$.

\section{Net Flow with No Oscillation}

For completeness Figure 11 shows the results of experimental axial dispersion measurements made using the method shown in case study 1 for experiments with net flow but no oscillation. This corresponds to regular pipe flow with a series of flow restrictions (the baffles).

For $R e_{\mathrm{n}}>400$ the measured axial dispersion increases proportionally and $E$ is approximately equal to $10^{-6} \times R e_{\mathrm{n}}$. Taking the fluid properties of water, the value of $E / U d \approx 1$ i.e., the measured axial dispersion is approximately half that expected for an open tube containing no baffles and turbulent flow for which it would be

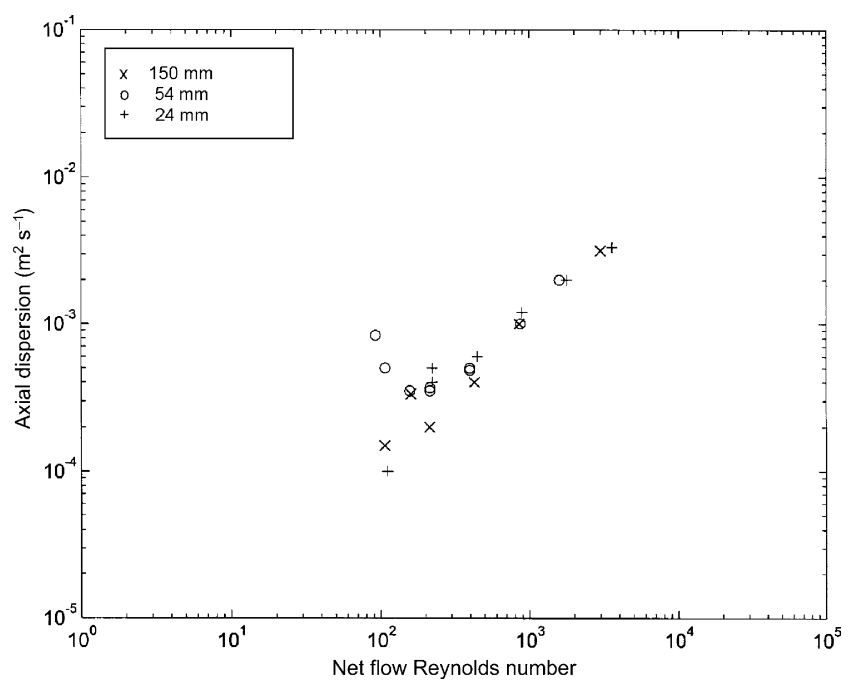

Figure 11. $E$ versus $R e_{\mathrm{n}}$ in the $24 \mathrm{~mm}, 54 \mathrm{~mm}$ and $150 \mathrm{~mm}$ apparatus with net flow only; $R e_{\mathrm{o}}=0$. expected that $E / U d \approx 2$. This serves as a useful check on the validity of the data and confirms that the presence of the baffles reduces the axial dispersion in the absence of oscillation.

For $R e_{\mathrm{n}}<400$ there are large discrepancies in the results for the three sets of apparatus. This is thought to be a reflection of the unreliability of vortex-formation at these low Reynolds number flows for a non-reversing flow (without oscillation). Under these conditions, non-oscillatory flow mixing appears to be susceptible to very small changes in net flow-rate, baffle orifice construction and possibly vibration from the lab surroundings all of which can affect vortex formation at the baffle orifice.

For $R e_{\mathrm{n}}<100$ there is little or no vortex formation and the diffusion equation does not adequately model over short distances what is essentially a Poiseuille-type flow. Under these conditions the axial dispersion cannot be quantified using the imperfect pulse technique.

\section{Study of Backmixing for OFM with Net Flow}

For the experiments described the optical sensor located upstream of the tracer injection point was used to record a measure of the proportion of dye tracer which travelled a distance of two baffle spacings upstream of the injection point. In this work the ratio of the area under the concentration-time profile at the upstream sensor to the average area under the downstream concentration-time profiles is expressed as a percentage and termed 'percentage backmixing'.

In Figure 12 percentage backmixing is plotted against the experimentally-determined axial dispersion for $R e_{\mathrm{n}}=107$ and a range of different oscillatory conditions. There is a clear, if slightly surprising, correlation between percentage backmixing and axial dispersion: there are two possible values of percentage backmixing for a given axial dispersion. These correspond to flow conditions on each side of the observed minimum plotted in Figure 6. For oscillatory Reynolds number greater than the minimum in axial dispersion ( $R e_{\mathrm{o}}>300$ for these net flow conditions), the increasing intensity of oscillation causes an increasing proportion of the tracer to disperse upstream of the injection point,

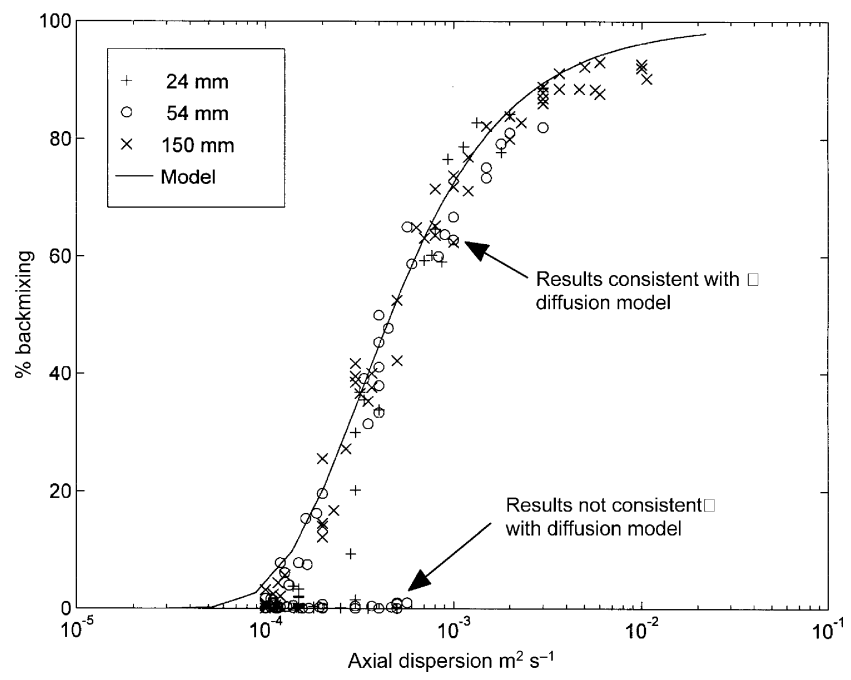

Figure 12. Percentage backmixing versus $E$ in the $24 \mathrm{~mm}, 54 \mathrm{~mm}$ and $150 \mathrm{~mm}$ apparatus; $R e_{\mathrm{n}}=107$. 
consequently increasing percentage backmixing. However, for oscillatory Reynolds numbers lower than the minimum in axial dispersion, the dispersion in the baffled tube is enlarged by the net flow component which serves to spread the tracer uni-directionally along the tube rather than providing an equal dispersion in both directions along the tube. Under these conditions there is insufficient driving force to cause tracer to move upstream of the injection point, and the percentage backmixing is zero.

There is broad agreement between diffusional model and experiment for non-zero values of percentage backmixing, with no adjustable parameters. However, the diffusion model theory does not predict the increasing axial dispersion when percentage backmixing $=0$. This is an interesting and useful result as the diffusion model is shown to be truly valid only when $R e_{\mathrm{o}}$ is greater than the value which corresponds to the minimum in axial dispersion; when $R e_{\mathrm{o}}$ is less than that value then the model can be used to quantify the axial dispersion downstream of the injection point, but cannot predict the upstream movement of the tracer.

In this way, a potentially useful method for determining the optimum oscillatory conditions necessary to minimize axial dispersion for a given net flow has been discovered: if the intensity of oscillations are increased until a small amount of tracer is mixed upstream after injection, (i.e., $0<$ percentage backmixing $<10 \%$ ) then the axial dispersion will be at or very close to its minimum value.

\section{Correlation for Axial Dispersion}

An empirical correlation was sought to describe axial dispersion as a function of the experimental flow conditions. Such a correlation would be useful for the estimation of residence time distributions for proposed baffled tube reactors and to help predict the likely effect on residence time distributions of modifying the net flow or oscillatory conditions.

Having established that axial dispersion is not a function of tube diameter, a range of experiments was performed using the $24 \mathrm{~mm}$ apparatus with varying oscillatory and net flow Reynolds values, the results of which are shown in Figure 13.

The data was matched to a general form of correlation proposed by (Crittenden et al., 1995). It was found that the power-law dependence of axial dispersion on Strouhal number proposed by (Crittenden et al., 1995) was inadequate to describe the variation in experimental measurements. Instead, these results suggested an exponential dependence of axial dispersion on Strouhal number.

The best-fit correlation was optimized by inspection and is superimposed on the graphs. The correlation with water as the fluid is therefore:

$$
\begin{aligned}
E= & 7.0 \times 10^{-7} \operatorname{Re}_{\mathrm{n}}^{0.8}+7.5 \times 10^{-7} \operatorname{Re}_{\mathrm{o}} e^{(-0.4 S t r)} \\
+ & \frac{3.0 \times 10^{-12} \operatorname{Re}_{\mathrm{n}}^{1.6}}{7.0 \times 10^{-7} \operatorname{Re}_{\mathrm{n}}^{0.8}+7.5 \times 10^{-7} \operatorname{Re}_{\mathrm{o}} e^{(-0.4 S t r)}} \\
& \left(\mathrm{m}^{2} \mathrm{~s}^{-1}\right)
\end{aligned}
$$

An interesting feature of the data not seen in Figure 13 is that in several cases, the measured axial dispersion for a given $R e_{\mathrm{o}}$ and $S t r$ is actually reduced by the presence of a small net flow. This is most unexpected since it would

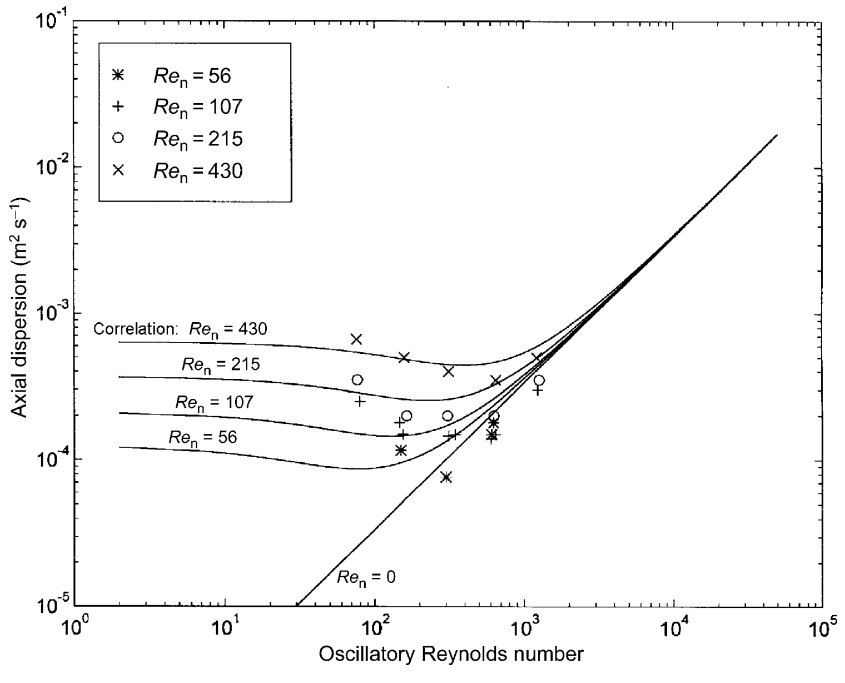

Figure 13. Graph showing a best-fit correlation for axial dispersion in the $24 \mathrm{~mm}$ apparatus in the case of net flow and oscillation $S t r=2$.

have been anticipated in practice that a net flow would if anything have served to increase the magnitude of the axial dispersion. The reduction is explained in the light of flow visualizations reported elsewhere (Smith, 1999) for which it appears that the introduction of a net flow can increase the degree of axisymmetry of the flow for oscillatory conditions that would otherwise give rise to asymmetric flow. By reducing the asymmetry in the flow the axial dispersion is also decreased since the flow is more ordered.

Using equation (10) and the arguments put forward by Crittenden et al. (1995) the oscillatory conditions for which the minimum axial dispersion occurs are when

$$
R e_{\mathrm{o}} e^{(-0.4 S t r)}=\frac{\left(\sqrt{A_{3}}-A_{1}\right)}{A_{2}} \operatorname{Re}_{\mathrm{n}}^{0.8}=1.37 R e_{\mathrm{n}}^{0.8}
$$

for which the predicted minimum axial dispersion is

$$
E=2 \sqrt{A_{3}} R e_{\mathrm{n}}^{0.8}=3.5 \times 10^{-6} \operatorname{Re}_{\mathrm{n}}^{0.8}\left(\mathrm{~m}^{2} \mathrm{~s}^{-1}\right)
$$

For example, in the case of $R e_{\mathrm{n}}=100$ and $S t r=1$ then the minimum value of axial dispersion is $1.4 \times 10 \mathrm{~m}^{2} \mathrm{~s}^{-1}$ and this occurs at an oscillatory Reynolds number of $R e_{\mathrm{o}}=82$. This prediction of the oscillatory Reynolds number which will give the minimum axial dispersion is slightly lower than would be expected from experimental experience: a more typical rule of thumb is that the oscillatory Reynolds number should be between two and five times the magnitude of the net flow Reynolds number in order to achieve minimum axial dispersion (Stonestreet, 1997).

\section{MULTI-ORIFICE BAFFLES AS A ROUTE TO SCALE-UP}

The preceding results provide strong evidence that for a given geometry of periodically-spaced single orifice baffles, axial dispersion in OFM is not a function of tube diameter for a given $R e_{\mathrm{n}}, R e_{\mathrm{o}}$ and Str. A practical difficulty with this method of scale-up is that with increasing tube 
diameter, the intensity and length-scale of mixing reduces as well as the opportunity for improved heat and mass transfer processes. An alternative solution to geometrical scaling-up of OFM was proposed by $(\mathrm{Ni}, 1994)$ who operated several $24 \mathrm{~mm}$ diameter tubes in parallel. This method has the advantages of predictable fluid mechanics and good heat-transfer capabilities, although a potentially vast number of individual tubes would be required for a large throughput process and there are potential issues of distributing feed and oscillations evenly to each tube. Scale-up and design of OFM for a wide range of potential applications was reviewed by (Ni et al., 2003).

An approach to the scale-up of OFM adopted in this work follows early work pioneered by Karr in relation to the perforated plate column and that has become a most established process using oscillatory flow in the chemical industry in general; a wide body of literature exists see for example (Karr et al., 1987) and (Baird and Rama Rao, 1998). Instead of having many small diameter tubes operating in parallel, the concept of a single large-diameter tube containing closely-spaced baffles each with many orifices was developed and is depicted in Figure 14. The design therefore mimics the effect of many smaller diameter tubes in parallel and appears similar to a reciprocating plate column (except that the fluid is oscillated and not the baffles). The predicted advantage of the multi-orifice baffles in the $150 \mathrm{~mm}$ diameter tube is that the same shear-rates and intensity of mixing can be achieved as in the $24 \mathrm{~mm}$ diameter tube while greatly increasing the throughput of the process. The $150 \mathrm{~mm}$ apparatus was adapted to take a $1 \mathrm{~m}$ long baffle insert with multi-orifice baffles. Each baffle was constructed from $1 \mathrm{~mm}$ thick PVC sheeting using a lathe and drill press and designed to push-fit the existing $150 \mathrm{~mm}$ diameter tube. The arrangement of orifices was essentially a two-dimensional hexagonal closepacked array of 37 holes (one in the middle, surrounded by rings of six, 12 and 18 orifices respectively), with slight modifications close to the tube wall in order to maximise the distance between each individual orifice and its neighbours. Each orifice had a diameter of $12 \mathrm{~mm}$ (the same as the baffle orifice diameter in the $24 \mathrm{~mm}$ apparatus).
A photograph of the multi-orifice baffle insert is shown in Figure 15.

Axial dispersion measurements were performed in the same manner as for the single-orifice baffled tubes. Dye tracer was injected via a cylinder of porous plastic that acted as a diffusive nozzle. The porous plastic allowed the dye to be injected quite rapidly into the tube while exiting evenly in all directions from the cylinder and with negligible momentum. A similar optical sensor arrangement was used: three sensors downstream of the injection point and one upstream, each spaced two inter-baffle cells from one another or from the dye injection point. The concentration-time profiles measured over a range of oscillatory conditions were typical of those obtained for single-orifice OFM. However it was found that the diffusion model did not provide a good model fit for downstream sensors 1 to 2 or 1 to 3 , but did give a good model fit for sensors 2 to 3 . This was thought to be an indication that at the position of sensor 1 (closest to the dye injection point) the dye had insufficient time to disperse radially across the diameter of the tube and was concentrated in the central region of the tube. By the time the dye had reached sensors 2 and 3, it was distributed sufficiently evenly radially across the tube for the axial diffusion model to be valid at the tube centre-line. In such cases the best-fit value of the axial dispersion coefficient obtained from sensors 2 and 3 was taken as the result and the data from sensor 1 was disregarded. For this reason also, percentage backmixing measurements were not considered reliable using a point injection technique and are therefore not presented. Estimates of the radial dispersion have been reported elsewhere (Smith, 1999).

In calculating the values of $R e_{\mathrm{n}}, R e_{\mathrm{o}}$ and $S t r$ it was clear that each term would need to be redefined to account for the change in geometry: the characteristic dimension $d$ (formerly the tube diameter) was replaced by $d_{\mathrm{e}}$ (equivalent diameter of a notional tube surrounding each orifice) which was the diameter equivalent to the total baffle area divided by the number of orifices. Within experimental error this was very close to an effective diameter of $24 \mathrm{~mm}$, hence the baffle spacing of $36 \mathrm{~mm}$ i.e., one-and-a-half

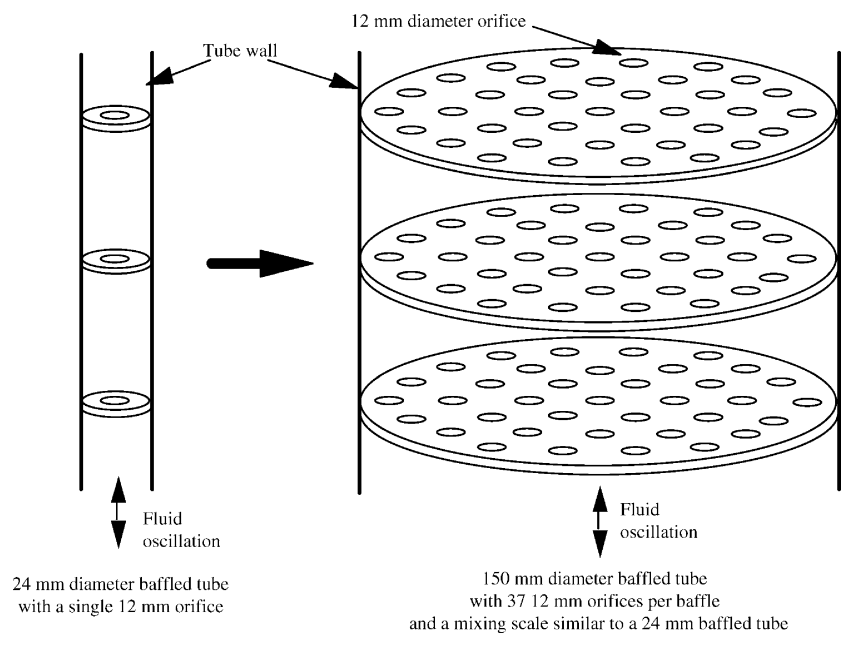

Figure 14. Using multi-orifice baffles to mimic many single-orifice baffled tubes in parallel.

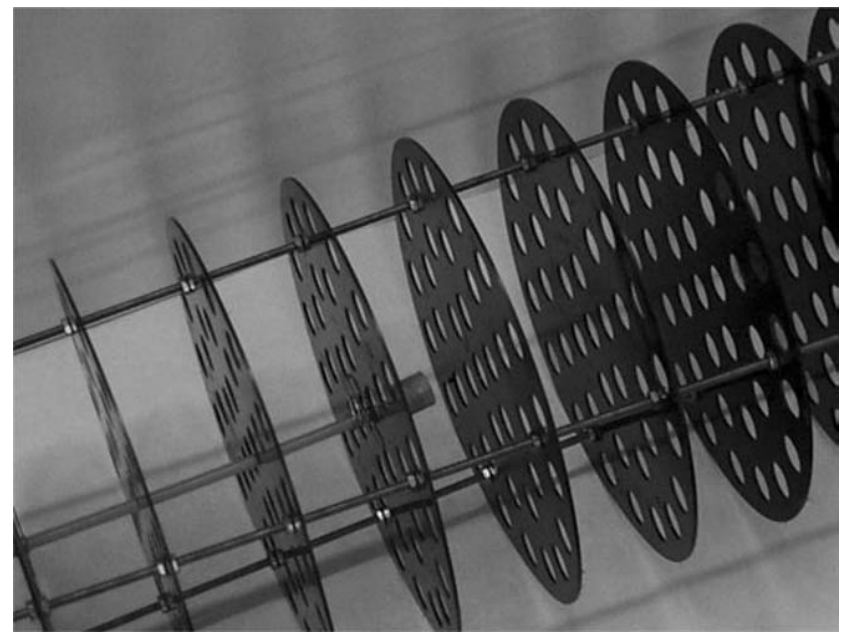

Figure 15. Multi-orifice baffle insert for the $150 \mathrm{~mm}$ apparatus (including dye injection tube). 


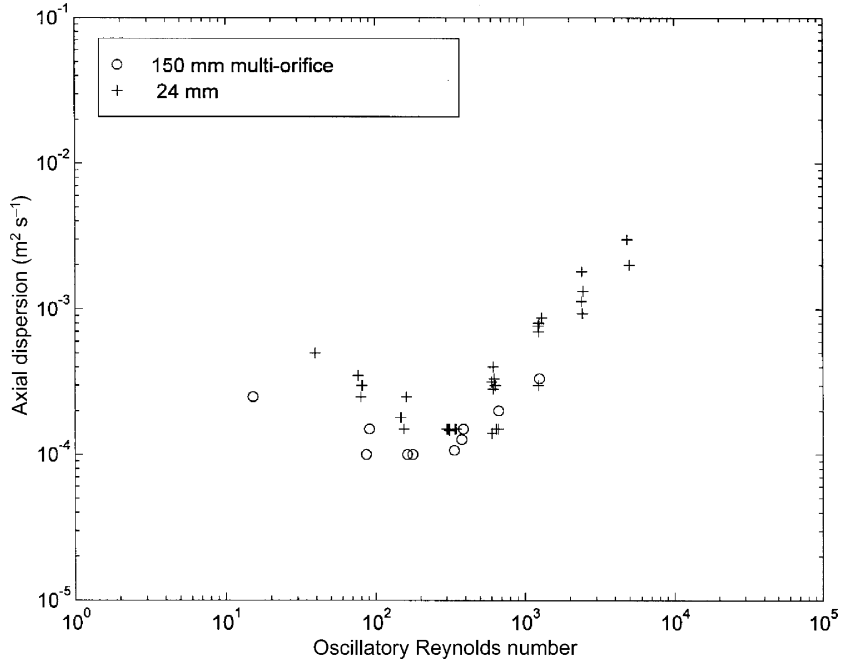

Figure 16. Axial dispersion measurements for multi-orifice baffles in the $150 \mathrm{~mm}$ apparatus compared to single-orifice baffles in the $24 \mathrm{~mm}$ apparatus for oscillations and net flow $R e_{\mathrm{n}}=107$ (Reynolds numbers for the multi-orifice baffles are based upon an equivalent tube diameter).

times the effective tube diameter. Figure 16 show the results of axial dispersion measurements with oscillations and net flow present. It can be seen that the measured values of axial dispersion for the multi-orifice baffles are very similar to those obtained for the single-orifice baffles. The range of conditions which could be investigated were limited for low $R e_{\mathrm{o}}$ by the validity of the diffusion model in interpreting the results, and for high $R e_{\mathrm{o}}$ by the flexibility of the multi-orifice baffles: for high intensity oscillations the baffles (being only $1 \mathrm{~mm}$ thick plastic and full of holes) began to flex considerably, despite the stainless steel supports providing a solid anchor-point. The use of stainless steel baffles and or a central baffle support would have reduced this problem.

It is noted that the multi-orifice baffles appear to give slightly lower values of axial dispersion for $R e_{\mathrm{o}}<200$. It is speculated that this could be due to the partial elimination of the stagnant corners behind the baffles which provided 'dead-zones' of flow when single-orifice baffles were investigated; with the multi-orifice baffles there is no tube wall between orifices therefore the potential for 'deadzones' is greatly reduced. For $R e_{\mathrm{o}}>200$ the differences in measured axial dispersion are insignificant because the vortices are anyway of sufficient strength to eliminate stagnant corners in the case of single-orifice baffles. Figure 17 shows measured values of axial dispersion in the $150 \mathrm{~mm}$ apparatus with single orifice and multi-orifice baffles. There is little difference in the results although there is relatively small range of overlap. Ni et al. (2001) related axial dispersion to several different column sizes and baffle geometries including variation of baffle spacing, hydraulic diameter, close-fit versus loose-fit and multiperforated plates for high $R e_{\mathrm{o}}$ conditions: this correlation provides a valuable tool for design of such systems.

The discovery that the use of multi-orifice baffles in place of single-orifice baffles does not substantially affect the measured axial dispersion is a key result of the work. It indicates that one can make laboratory-scale measurements of axial dispersion (e.g., in a $24 \mathrm{~mm}$ diameter baffled tube) and then use those results directly to predict with

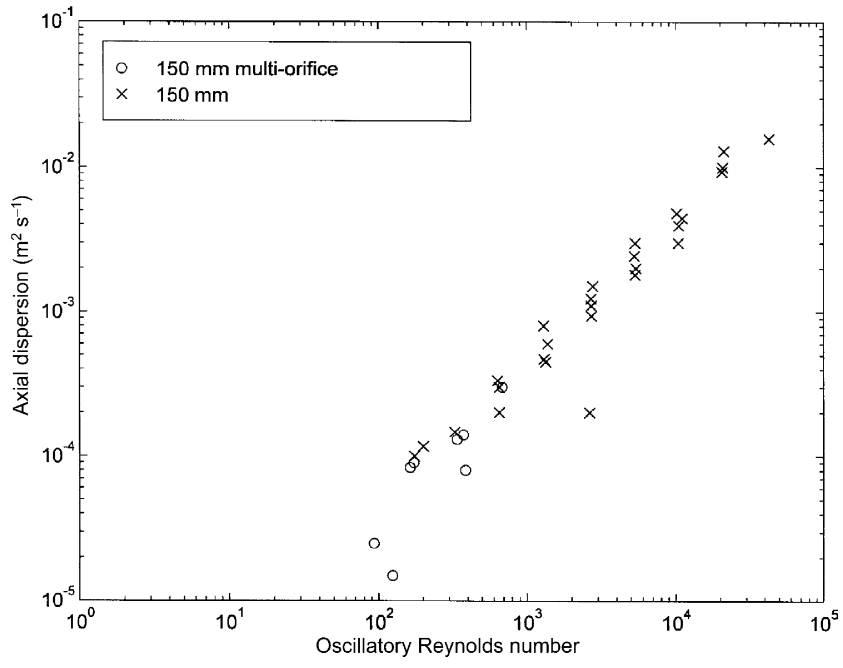

Figure 17. Axial dispersion measurements for multi-orifice baffles in the $150 \mathrm{~mm}$ apparatus compared to single-orifice baffles in the $150 \mathrm{~mm}$ apparatus for oscillatory flow only (no net flow).

confidence the magnitude of axial dispersion which will occur in a very much larger diameter tube using multi-orifice baffles (of equivalent geometry to the $24 \mathrm{~mm}$ tube). This could prove to be a significant advantage compared with the difficulties of, say, predicting the dispersion properties of a scaled-up continuous stirred tank reactor. Analagous systems described in the literature are discussed in the Handbook of Solvent Extraction, 1983 in which Simons reviewed data for pulsed packed colums (Ch. 11.1) and concluded that the scale-up factor for column height (in terms of column diameter) was unity since the flow pattern was heavily controlled by the packing; Logsdail \& Slater reviewed data on pulsed columns with perforated plates (Ch. 11.2) and indicated that axial dispersion increased with column diameter under some conditions.

\section{CONCLUSIONS}

The presentation of axial dispersion data measured in each of the $24 \mathrm{~mm}, 54 \mathrm{~mm}$ and $150 \mathrm{~mm}$ sets of apparatus has shown that the absolute magnitude of axial dispersion is not a function of tube diameter if the values of $R e_{\mathrm{n}}$, $R e_{\mathrm{o}}$ and $\mathrm{Str}$ are kept constant. An approximate correlation has been fitted to the experimental data. It has been discovered through the examination of the degree of backmixing of the injected dye tracer upstream of the injection point that the axial diffusion model is only truly applicable when the oscillatory Reynolds number is greater than that which gives the minimum measured axial dispersion. The diffusion model is therefore limited in the range of flow conditions for which it can be used to quantify experimental axial dispersion measured over short tube lengths.

The multi-orifice design appears particularly attractive because of the potential ease of manufacture for large plant and also because of the similarity of the observed fluid mechanics and axial dispersion between single and multi-orifice baffle designs. This means that in principle laboratory scale experiments could be carried out and the results be used with reasonable confidence to predict 
axial dispersion (and mixing intensity) in a very much larger industrial scale multi-orifice baffle reactor.

\section{NOMENCLATURE}

$\begin{array}{ll}C_{1}{ }^{\prime} & \text { normalized experimental concentrations at optical sensor } 1 \\ d & \text { tube internal diameter } \\ d_{\mathrm{e}} & \text { equivalent diameter } \\ E & \text { axial dispersion coefficient } \\ L & \text { length of tube } \\ Q & \text { net volumetric flow rate } \\ U & \text { mean net flow velocity through tube } \\ V & \text { volume of fluid between probes } \\ x_{\mathrm{o}} & \text { oscillatory amplitude (centre to peak) } \\ \mu & \text { viscosity of fluid } \\ \rho & \text { density of fluid } \\ \omega_{\mathrm{o}} & \text { angular velocity of oscillation }\end{array}$

\section{REFERENCES}

Baird, M.H.I. and Rama Rao, N.V., 1996, Hydrodynamics of reciprocating plate columns, 2nd Conference on Pulsatile Flow, 3 June, University of Strathclyde, Scotland.

Baird, M.H.I. and Rama Rao, N.V., 1998, Axial mixing in a $15 \mathrm{~cm}$ diameter reciptrocating plate bubble column, Can J Chem Eng, 76: 370-378

Brunold, C.R., Hunns, J.C.B., Mackley, M.R. and Thompson, J.W., 1989 , Experimental observations on flow patterns and energy losses for oscillatory flow in ducts containing sharp edges, Chem Eng Sci, 44(5): $1227-1244$.

Crittenden, B.D., Field, R.W. and Pervez, M.I., 1995, Oscillatory flow in packed beds and baffled tubes. A unifiying approach to the interpretation of experimental data, Chem Eng Sci, 50(23): 3839-3845.

Dickens, A.W., Mackley, M.R. and Williams, H.R., 1989, Experimental residence time distribution measurements for unsteady flow in baffled tubes, Chem Eng Sci, 44(7): 1471-1479.

Gilibaro, L.G., 1978, On the residence time distribution for systems with open boundaries, Chem Eng Sci, (33): 487-492.

Goebel, J.C., Booij, K. and Fortuin, J.M.H., 1986, Axial dispersion in single-phase flow in pulsed packed columns, Chem Eng Sci, 41(12): 3197-3203.

Handbook of Solvent Extraction, 1983 (Wiley-Interscience).

Howes, T., 1988, On the dispersion of unsteady flow in baffled tubes, $\mathrm{PhD}$ thesis, University of Cambridge.

Karr, A.E., Ramanujam, S., Lo, T.C. and Baird, M.H.I., 1987, Axial mixing and scaleup of reciprocating plate extraction columns, Can $J$ Chem Eng, 65: 373-381.
Levenspiel, O. and Smith, W.K., 1957, Notes on the diffusion type model for the longitudinal mixing of fluids in flow, Chem Eng Sci, 6: 227-233.

Mackley, M.R., 1987, Using oscillatory flow to improve performance, Chemical Engineer, February edition: 18-20.

Mackley, M.R., 1991, Process innovation using oscillatory flow within baffled tubes, Trans IChemE, (69): 197-199.

Mackley, M.R. and Ni, X., 1991, Mixing and dispersion in a baffled tube for steady laminar and pulsatile flow, Chem Eng Sci, 46(12): 3139-3151.

Mackley, M.R., Smith, K.B. and Wise, N.P., 1993, The mixing and separation of particle suspensions using oscillatory flow in baffled tubes, Trans IChemE Part A, (71): 649-656.

Mackley, M.R. and Ni, X., 1993, Experimental fluid dispersion measurements in periodic baffled tube arrays, Chem Eng Sci, 48(18): 3293-3305.

Mackley, M.R. and Stonestreet, P., 1995, Heat transfer and associated energy dissipation for oscillatory flow in baffled tubes, Chem Eng Sci, 50(14): 2211-2224.

$\mathrm{Ni}, \mathrm{X} ., 1994$, Residence time distribution measurements in a pulsed baffled tube bundle, J Chem Tech Biotechnol, (59): 213-221.

Ni, X., de Gélicourt, Y.S., Baird, M.H.I. and Rama Rao, N.V., 2001, Scaleup of single phase axial dispersion in batch and continuous oscillatory baffled tubes, Can J Chem Eng, 79: 444-448.

$\mathrm{Ni}, \mathrm{X}$. and Gough, P. 1997, On the discussion of the dimensionless groups governing oscillatory flow in a baffles tube, Chem Eng Sci, 52(18): 3209-3212.

Ni, X., Mackley, M.R., Harvey, A.P., Stonestreet, P., Baird, M.H.I. and Rama Rao, N.V., 2003, Mixing through oscillations and pulsations-a guide to achieving process enhancements in the chemical and process industries, Trans IChemE Part A, (81): 373-383.

Smith, K.B., 1999, The scale-up of oscillatory flow mixing, PhD thesis, University of Cambridge.

Stonestreet, P., 1997, private communication.

Westerterp, K.R., van Swaaij, W.P.M. and Beenackers, A.A.C.M., 1984, Chemical Reactor Design and Operation (John Wiley and Sons).

\section{ACKNOWLEDGEMENTS}

We would like to thank EPSRC for financial support, Dr Paul Stonestreet, currently of GSK Ltd Tonbridge UK, for technical advice at various stages of the project and also Prof Malcolm Baird of McMaster University Canada for review and technical advice.

The manuscript was received 8 March 2005 and accepted for publication after revision 28 July 2006. 